\title{
A Randomized, Two-Way Crossover Study to Evaluate the Pharmacokinetics of Caffeine Delivered Using Caffeinated Chewing Gum Versus a Marketed Caffeinated Beverage in Healthy Adult Volunteers
}

\author{
Paul Sadek, PhD, ${ }^{1}$ Xiao Pan, PhD, ${ }^{1}$ Phil Shepherd, PhD, ${ }^{1}$ Elise Malandain, DVM, MSc, ${ }^{1}$ \\ John Carney, $\mathrm{PhD},{ }^{2}$ and Hugh Coleman, $\mathrm{MD}^{3}$
}

Background: This study was conducted to compare the pharmacokinetics of caffeine delivered using caffeinated chewing gum to that delivered using a marketed caffeinated beverage (instant coffee) in 16 healthy adult volunteers.

Materials and Methods: This was a controlled open-label, randomized, two-period crossover study. Caffeinated chewing gum and a serving of instant coffee, each containing $\sim 50 \mathrm{mg}$ caffeine, were administered with blood samples collected before and up to 24 hours after administration starts. Plasma caffeine levels were analyzed using validated liquid chromatography coupled with tandem mass spectrometry methodology.

Results: There were no statistical differences between the two caffeine products in $t_{\max }(p=0.3308)$ and $k_{\mathrm{a}}(p=$ 0.3894 ). Although formulated at $\sim 50 \mathrm{mg}$ caffeine each, mean dose released from chewing gum was $\sim 18 \%$ less than beverage. Dose-normalized area under the concentration-time curve $(\mathrm{AUC})_{0-\mathrm{t}}, \mathrm{AUC}_{0-\infty}$, and $C_{\max }$ was similar between products. Although the criteria were not set a priori and the study was not powered for concluding bioequivalence, the $90 \%$ confidence intervals fell within the bioequivalence limit of $80 \%$ to $125 \%$. Conclusions: Existing scientific literature on caffeine, based mostly on data from caffeinated beverages, can be leveraged to support the safety of caffeine delivered by chewing gum and current maximum safe caffeine dose advice should be applicable irrespective of delivery method.

Keywords: caffeine, chewing gum, pharmacokinetics, relative bioavailability

\section{Introduction}

C AFFEINE IS THE most commonly consumed stimulant in the world ${ }^{1,2}$ and is often the chosen means to maintain alertness and counteract the effects of sleep deprivation and fatigue. At plasma concentrations achieved after ingestion of common dietary sources, caffeine exerts its psychoactive effects through blockade of the A1 and A2 adenosine receptors in the brain, promoting the release of a range of neurotransmitters that positively affect cognitive function. ${ }^{3-6}$ Studies have demonstrated favorable effects of caffeine on arousal state (attention, alertness, and reaction time) at doses from 32 to $300 \mathrm{mg}^{5}$ Effects are generally dose dependent, with low doses associated with improvements in mood and a reduction in anxiety, especially in highly fatigued subjects, while high doses may overstimulate, leading to difficulty falling asleep and increased anxiety and nervousness. ${ }^{7}$

With caffeine so widely available and consumed, the impact on consumer health has been extensively studied. Side effects of excessive caffeine intake may include anxiety, agitation, restlessness, and insomnia. ${ }^{6}$ Single doses of up to $200 \mathrm{mg}$ caffeine are not considered to give rise to safety concerns $^{8}$ and consumption of $400 \mathrm{mg}$ caffeine per day is generally safe in healthy adults, not being associated with any adverse cardiovascular, behavioral, reproductive,

\footnotetext{
${ }^{1}$ Mars Wrigley Confectionery, Chicago, Illinois.

${ }^{2}$ JMC Consulting, Portland, Oregon.

${ }^{3}$ Covance Daytona Clinical Research Unit, Inc., Daytona Beach, Florida.

(c) Paul Sadek et al. 2017; Published by Mary Ann Liebert, Inc. This article is available under the Creative Commons License CC-BY-NC (http://creativecommons.org/licenses/by-nc/4.0). This license permits non-commercial use, distribution and reproduction in any medium, provided the original work is properly cited. Permission only needs to be obtained for commercial use and can be done via RightsLink.
} 
acute, or bone status effects. ${ }^{2}$ In pregnant women, metabolism of caffeine is altered and effects are longer lasting; current evidence suggests that daily doses of $200 \mathrm{mg}^{8}$ to $300 \mathrm{mg}^{2}$ in healthy pregnant or lactating women do not pose safety concerns for the developing fetus or infant.

The vast majority of studies investigating the safety of caffeine, upon which the above conclusions are based, were conducted following exposure to known doses of caffeine administered as food, beverages, or oral medications for which the kinetics of intestinal absorption are well characterized. Caffeine is absorbed rapidly and essentially completely from the small intestine within an hour of ingestion ${ }^{9}$ and diffuses rapidly into other tissues, including the brain, with the maximum observed plasma concentration at $\sim 0.5$ to 2 hours postdose. ${ }^{10}$ The pharmacokinetics $(\mathrm{PK})$ has been shown to be dose proportional at doses up to $750 \mathrm{mg} .{ }^{11-13}$ Caffeine is predominantly metabolized by cytochrome p450 (CYP) 1A2 in the liver and has a half-life of $\sim 2$ to 12 hours, with sources of variation including concomitant smoking, food intake, disease, drug usage, and pregnancy, as well as gastric emptying speed. ${ }^{6}$

Recently, new formulations for delivery of caffeine have been developed, including caffeine-containing chewing gum, which represent a convenient and portable source. ${ }^{14,15}$ However, because administration of caffeine by gum also could entail the absorption of caffeine through the mucosal membranes of the mouth, the PK of which is less well characterized compared with intestinal absorption, it is unclear whether the safety findings of specific doses delivered by ingestion may be applied to equivalent doses delivered by gum. Absorption through the buccal cavity may be increased due to the high level of vascularization and lymphatic drainage compared to the intestine, combined with the avoidance of first-pass metabolism and presystemic elimination. ${ }^{16}$ Actual exposure to caffeine contained in gum may also be influenced by factors such as the rate of release from the gum, chew rate, and saliva production. ${ }^{14,15}$

This study compares the PK of caffeine administered as a chewing gum pellet containing $\sim 50 \mathrm{mg}$ caffeine with that following consumption of a marketed caffeinated beverage (instant coffee) at approximately the same strength per serving.

\section{Materials and Methods}

\section{Study population}

The study was conducted at Covance Clinical Research Unit, Inc. (Daytona Beach, Florida), in accordance with the standards of the International Council for Harmonisation guidelines, commonly known as Good Clinical Practices, which are consistent with the Declaration of Helsinki. The protocol, protocol amendment, advertisement, and informed consent forms were reviewed and approved by Midlands Independent Review Board (Overland Park, KS). A written consent form was signed by each enrolled subject.
A total of 16 subjects met the study screening criteria and were enrolled in the study (see Study Design and Treatments section below). The design was set up to obtain an initial comparison of PK of caffeine from a chewing gum and beverage. Any statistically significant difference could then be used to power a study, if desired, to test the significance of statistical difference. All subjects were required to be nonsmoking healthy males or females between the ages of 18 to 50 years with a body mass index of 18.0 to $29.9 \mathrm{~kg} / \mathrm{m}^{2}$. Subjects were in good health as determined by the absence of clinically significant history or clinical manifestation of any metabolic, allergic, dermatological, hepatic, renal, hematological, pulmonary, cardiovascular, gastrointestinal, neurological, or psychiatric disorders, or clinically relevant findings from 12-lead electrocardiograms (ECGs), vital signs, physical examination, and clinical laboratory tests. Females were required to be nonpregnant, nonlactating, or postmenopausal for at least 1 year or surgically sterile for at least 90 days at the time of screening. All premenopausal females were required to have a negative pregnancy test. Restrictions were placed on the use of prescription medications, hormonal contraceptives, and over-the-counter nonprescription medications. Subjects were required to have a history of caffeine consumption and had to be willing to chew gum and drink black coffee. In addition, subjects had to be willing to abstain from consuming caffeine-containing food or beverages from the time of check-in (day -2) until discharge (day 4), except for study products provided by qualified clinical staff during confinement at the clinical site.

\section{Study design and treatments}

This single-center, open-label, randomized, twoperiod crossover, controlled clinical study compared the PK and safety and tolerability profiles of a new caffeinated chewing gum product to a currently marketed caffeinated beverage (instant coffee), each containing $\sim 50 \mathrm{mg}$ caffeine (see investigational product details below). Before the administration of the first caffeine product in Period 1 (day 1), subjects were randomly assigned to one of two treatment sequences (test product followed by reference product or reference product followed by test product). With a crossover design, subjects received a single dose of each of the two caffeine products, chewing gum and beverage, with a washout period of $\sim 48$ hours between product administrations. Following the administration of each caffeinated product, blood samples were collected at specific time points and used to analyze the PK profiles of the caffeine products. Each caffeine product was administered to subjects in a fasted state on day 1 (Period 1) and day 3 (Period 2). Caffeine products were administered to subjects following an overnight fast of at least 10 hours from food (not including water) and each administration was followed by a fast from food (not including water) for at least 2 hours. In 
addition, subjects were required to consume $\sim 240 \mathrm{~mL}$ ( $8 \mathrm{fl}$. oz.) of water within 5 minutes before the administration of each product.

The investigational product (test product), pellets of caffeinated chewing gum (Lot No. 081315 SMA0020 80008; CSPC Pharmaceutical Group Limited), was manufactured and supplied to the clinical site by the Sponsor (Mars Wrigley Confectionery, Chicago, IL). Subjects were required to chew the gum for 10 minutes, after which the subject discarded the gum. The chewed gum cud was collected and analyzed for the remaining caffeine content by the Sponsor. The amount of caffeine remaining in the gum cud was used to calculate the actual dose of caffeine released during chewing for each subject. The caffeinated beverage (instant coffee; Lot No. 5230209120036) was prepared at the clinical site and was dispensed at a temperature of $125^{\circ} \mathrm{F}\left(52^{\circ} \mathrm{C}\right)$ $\pm 5^{\circ} \mathrm{F}\left(3^{\circ} \mathrm{C}\right)$. Subjects were required to consume a $4 \mathrm{fl}$. oz. cup of the prepared coffee within a timeframe of 1 minute or less. Samples of the caffeinated beverage were collected and analyzed for caffeine content by Covance Laboratories, Inc. (Madison, WI). The estimated caffeine content administered by the single serving of prepared coffee was used to calculate the actual caffeine dose each subject received from the caffeinated beverage.

Safety was assessed at specific time points during the study and included the incidence of adverse events (AEs), clinical laboratory tests, vital signs, 12-lead ECGs, and physical examinations.

\section{Sampling and analytical procedures}

Blood samples $(6 \mathrm{~mL})$ were drawn through an intravenous catheter and/or direct venipuncture and collected into $\mathrm{K}_{2}$ EDTA Vacutainer ${ }^{\circledR}$ tubes on day 1 and 3 before the administration of each product ( $\sim 10$ minutes before product use); at 5, 10, 15, 20, 25, 30, 35, 40, 45, 50, 60, and 90 minutes from the start of gum chewing or coffee drinking; and at 2, 3, 4, 6, 8, 10, 12, 16, and 24 hours from the start of gum chewing or coffee drinking. After separation at $\sim 2000 \mathrm{~g}$ for 15 minutes at $\sim 5^{\circ} \mathrm{C}$, duplicate plasma samples were transferred into labeled storage tubes and were immediately frozen and stored in a freezer at $-20^{\circ} \mathrm{C}$ or below until analyzed. Plasma concentrations of caffeine were determined by Covance Laboratories, Inc., using a validated liquid chromatography coupled with tandem mass spectrometry (LC-MS/MS) method. ${ }^{17}$ Plasma samples were spiked with internal standards (caffeine-d9 and paraxanthine 1-methyl d3), extracted from human plasma by solid-phase extraction, and analyzed using LC-MS/MS. The chromatography was performed using an LC-MS/MS (API 4000 ${ }^{\mathrm{TM}}$, operated in positive electrospray ionization [ESI+] mode; SCIEX) system and was controlled using Analyst ${ }^{\circledR}$ (SCIEX) software. The lower limit of quantification for caffeine was $25.0 \mathrm{ng} / \mathrm{mL}$.

\section{Pharmacokinetic and statistical analysis}

The sample size for this study was based upon precedent set by other PK studies of similar nature and was not based on power calculations. Fourteen evaluable subjects (seven subjects per treatment sequence) were considered sufficient to adequately characterize the PK profile of caffeine and obtain reliable estimates and $90 \%$ confidence intervals (CIs) for the mean ratios of the PK parameters between caffeine delivered by caffeinated chewing gum and a marketed caffeinated beverage.

Noncompartmental and compartmental modeling were used to determine caffeine PK parameters following the administration of each product. ${ }^{18}$ The concentrationtime data were evaluated using Phoenix WinNonlin ${ }^{\circledR}$ (Version 6.2.1; Certara USA, Inc.). The maximum observed concentration $\left(C_{\max }\right)$ and the time to reach $C_{\max }$ $\left(t_{\max }\right)$ were obtained from the concentration-time profile. The area under the concentration-time curve (AUC) from hour 0 to the time of the last measurable concentration $\left(\mathrm{AUC}_{0-\mathrm{t}}\right)$ was determined by the trapezoidal method. The AUC extrapolated to infinity $\left(\mathrm{AUC}_{0-\infty}\right)$ was calculated using the following equation:

$$
\mathrm{AUC}_{0-\infty}=\mathrm{AUC}_{0-\mathrm{t}}+C_{\mathrm{t}} / \lambda_{\mathrm{z}}
$$

where $C_{\mathrm{t}}$ is the last measureable concentration and $\lambda_{\mathrm{z}}$ is the apparent terminal elimination phase constant. The $\lambda_{\mathrm{z}}$ was determined by linear regression of the $\log$ concentration versus time profile during the terminal phase. The elimination half-life $\left(t_{1 / 2}\right)$ was estimated from the natural $\log 2 / \lambda_{\mathrm{z}}$. Dose-normalized (DN) values for $\mathrm{AUC}_{0-\mathrm{t}}, \mathrm{AUC}_{0-\infty}$, and $C_{\max }$ were calculated by dividing the calculated PK parameters by the actual received dose (mg). Absorption rate constant $\left(k_{\mathrm{a}}\right)$ for caffeine following administration of each product was calculated using compartmental modeling.

Relative bioavailability was evaluated between caffeine released by caffeinated chewing gum (test product) versus that administered by a marketed caffeinated beverage (reference product). The mixed-model procedure was used and included sequence, period, and study product as fixed effects and subject within sequence as a random effect. Data for $\mathrm{DN} \mathrm{AUC}_{0-\mathrm{t}}, \mathrm{DN} \mathrm{AUC}_{0-\infty}$, and $\mathrm{DN} C_{\max }$ were natural log-transformed before analysis. The point estimate of the geometric mean ratio (GMR) of test product relative to reference product and corresponding 90\% CI of the GMR for these PK parameters were provided for the comparison between test and reference products. Wilcoxon's signedrank test was used to assess the difference in $t_{\max }$ and $k_{\mathrm{a}}$ between test and reference products. Statistical analyses were conducted using SAS $^{\circledR}$ Version 9.3.

\section{Results}

\section{Demographics}

Sixteen subjects ( 8 males and 8 females) were enrolled and randomized to one of two treatment sequences and 
all 16 subjects completed the study. The mean (standard deviation [SD]) age was 35 [9.7] years with a range of 21 to 47 years. Mean (SD) body mass index was 26.0 (2.50) $\mathrm{kg} / \mathrm{m}^{2}$ with a range of 21.5 to $29.3 \mathrm{~kg} / \mathrm{m}^{2}$. The majority of the subjects were white $(68.8 \%), 18.8 \%$ were black or African American, and $12.5 \%$ were classified as of other races.

\section{Pharmacokinetic results}

Each of the 16 subjects received a single dose of $\sim 50 \mathrm{mg}$ caffeine using caffeinated chewing gum (test product) and a single dose of $\sim 50 \mathrm{mg}$ caffeine using caffeinated beverage (reference product). Based on the FDA guidance for assessing bioequivalence, ${ }^{19}$ one subject was excluded from the descriptive statistical analyses for DN $\mathrm{AUC}_{0-\mathrm{t}}, \mathrm{DN} \mathrm{AUC}_{0-\infty}$, and DN $C_{\max }$ of caffeinated chewing gum because the quantifiable predose value before Period 2 dosing was greater than $5 \%$ of the $C_{\max }$ value, but was included in the descriptive statistical analyses for the caffeinated beverage. This subject was excluded from the statistical analyses for $t_{\max }$ and $k_{\mathrm{a}}$ of both the caffeinated chewing gum and caffeinated beverage.

A summary of caffeine exposure (actual dose) following administration of the two caffeine products is presented in Table 1. Mean ( \pm SD) actual caffeine doses released by the caffeinated chewing gum were 40.81 $( \pm 3.206) \mathrm{mg}$ (range of 34.07 to $48.41 \mathrm{mg}$ ) compared to $50.65( \pm 0.496) \mathrm{mg}$ (range of 49.88 to $51.39 \mathrm{mg}$ ) delivered by the caffeinated beverage. The mean actual dose of caffeine released by the caffeinated chewing gum was $\sim 18 \%$ less than the planned dose of $50 \mathrm{mg}$ caffeine, whereas the mean actual dose delivered by the caffeinated beverage was, approximately, as planned. This is because, about $18 \%$ of the caffeine was not released from the gum matrix during chewing.

Arithmetic mean $( \pm S D)$ caffeine plasma concentration versus time profiles following administration of a single dose of $\sim 50 \mathrm{mg}$ caffeine by caffeinated chewing gum $(n=15)$ and a marketed caffeinated beverage $(n=16)$ through 120 minutes after caffeine administration and through 24 hours after caffeine administration are pre-

Table 1. Summary of Caffeine Exposure (ACTUAL Doses)

\begin{tabular}{lcc}
\hline & \multicolumn{2}{c}{ Planned caffeine doses } \\
\cline { 2 - 3 } Statistic & $\begin{array}{c}\text { Caffeinated chewing } \\
\text { gum 50 mg }\end{array}$ & $\begin{array}{c}\text { Caffeinated } \\
\text { beverage 50 mg }\end{array}$ \\
\hline $\begin{array}{c}\text { Actual mean } \\
\text { dose (mg) }\end{array}$ & 40.81 & 50.65 \\
SD & 3.206 & 0.496 \\
Median & 40.62 & 50.45 \\
Min., max. & $34.07,48.41$ & $49.88,51.39$ \\
$n$ & 16 & 16 \\
\hline
\end{tabular}

$n$, number of subjects; SD, standard deviation. sented in Figures 1 and 2, respectively. The corresponding summary statistics of the PK parameters for caffeine following administration of a single dose of $\sim 50 \mathrm{mg}$ caffeine using caffeinated chewing gum and a marketed caffeinated beverage are presented in Table 2. Formal statistical analyses of the PK parameters for caffeine are presented in Table 3.

Absorption was rapid following administration of both caffeine products with median (minimum to maximum) $t_{\max }$ values of 0.667 (0.250 to 1.50$)$ hours following caffeine administration using the caffeinated chewing gum and 0.625 ( 0.333 to 1.50$)$ hours following the caffeinated beverage. The median (minimum to maximum) lag time to the first quantifiable concentration was 0 (range of 0 to 0.0833 ) hours for both products. The geometric mean values for $k_{\mathrm{a}}$ were similar with values of 2.99 and 2.72 hours $^{-1}$ for caffeinated chewing gum and caffeinated beverage, respectively. The median of difference (90\% CI) for $t_{\max }$ and $k_{\mathrm{a}}$ for the caffeinated chewing gum to the caffeinated beverage were $-0.08(-0.17,0.08)$ and 0.33 $(-0.36,1.15)$, respectively (Table 3$)$. The statistical analyses show no statistical differences $(p \geq 0.05)$ between the caffeinated chewing gum and the caffeinated beverage in $t_{\text {max }}(p=0.3308)$ or $k_{\mathrm{a}}(p=0.3894)$.

After reaching $C_{\max }$, caffeine concentrations readily declined in a generally monophasic manner (Fig. 2). Mean (SD) $t_{1 / 2}$ values were similar between the two products with values of 4.91 (1.44) hours and 4.70 (1.26) hours following administration of the caffeinated chewing gum and caffeinated beverage, respectively.

The geometric mean non-DN $\mathrm{AUC}_{0-\mathrm{t}}, \mathrm{AUC}_{0-\infty}$, and $C_{\max }$ were approximately $14 \%, 13 \%$, and $9 \%$ lower for the caffeinated gum than the caffeinated beverage $\left(\mathrm{AUC}_{0-\mathrm{t}}, \mathrm{AUC}_{0-\infty}\right.$, and $C_{\max }$ values of $6990 \mathrm{~h} \cdot \mathrm{ng} / \mathrm{mL}$, $7440 \mathrm{~h} \cdot \mathrm{ng} / \mathrm{mL}$, and $1180 \mathrm{ng} / \mathrm{mL}$, respectively, for the caffeinated chewing gum and $8100 \mathrm{~h} \cdot \mathrm{ng} / \mathrm{mL}, 8520 \mathrm{~h} \cdot \mathrm{ng} / \mathrm{mL}$, and $1300 \mathrm{ng} / \mathrm{mL}$, respectively, for the caffeinated beverage). As noted earlier, the mean caffeine dose was $\sim 18 \%$ lower for the caffeinated chewing gum than the caffeinated beverage.

Relative bioavailability was examined between caffeine administered using the caffeinated chewing gum and caffeinated beverage. Table 3 shows the statistical analysis of the DN PK parameters $\mathrm{AUC}_{0-\mathrm{t}}, \mathrm{AUC}_{0-\infty}$, and $C_{\max }$, comparing caffeine exposure following the administration of caffeinated chewing gum and caffeinated beverage. Based on the ratios of geometric least-squares (LS) means for the DN $\mathrm{AUC}_{0-\mathrm{t}}, \mathrm{AUC}_{0-\infty}$, and $C_{\max }$, caffeine exposure was $\sim 7 \%$ to $12 \%$ higher following administration of the caffeinated chewing gum compared to the caffeinated beverage.

\section{Adverse events and tolerability profile}

The two caffeine products were well tolerated by subjects in this study. There were no discontinuations due to AEs for any of the healthy subjects participating in this 


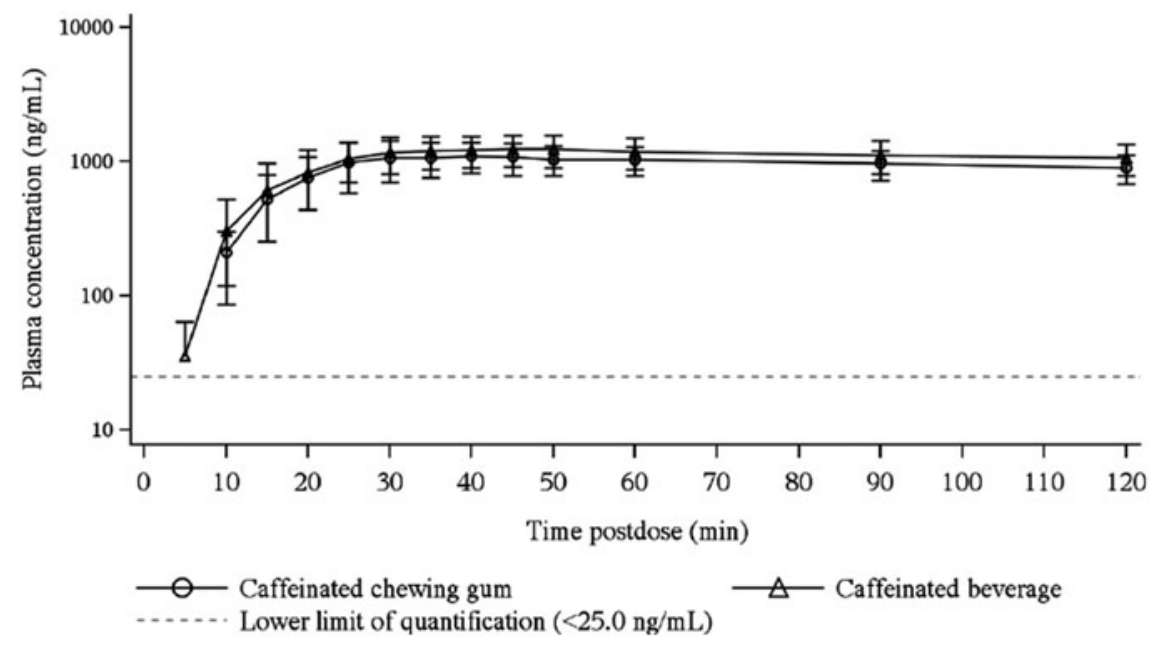

FIG. 1. Arithmetic mean caffeine plasma concentration versus time (through 120 minutes) in semilogarithmic scale following administration of a single dose of $\sim 50 \mathrm{mg}$ caffeine using caffeinated chewing gum $(n=15)$ and a marketed caffeinated beverage $(n=16)$ to healthy subjects.

study. A total of two AEs (near syncope and leg pain) were reported during the study. Following the administration of caffeinated chewing gum, one subject experienced near syncope, which occurred $\sim 16$ hours after administration of the caffeinated chewing gum, followed by leg pain $\sim 23$ hours after dose administration. Both events were recorded as mild in intensity and resolved by the end of the study. Near syncope was considered not applicable to product administration and leg pain was considered unlikely to be related to the product. Overall, there were no clinically relevant findings in clinical laboratory evaluations, vital signs, 12-lead ECGs, or physical examinations during the study and no differences were observed in the tolerability profile between the two caffeine products.

\section{Discussion}

The objective of this study was to compare the PK of a single 50-mg dose of caffeine after delivery using caffeinated chewing gum (test product) or a marketed instant cof- fee (reference product) in healthy adult volunteers. The results indicate that there is no marked difference in the PK profile of caffeine delivered by the two products. While the differences in exposure (DN $\mathrm{AUC}_{0-\mathrm{t}}, \mathrm{AUC}_{0-\infty}$, and $C_{\max }$ ) might suggest a more efficient absorption of the caffeine from the gum, the differences were not significantly different between the delivery methods and the $90 \%$ CIs for the ratios of AUC, and $C_{\max }$ fell within the recommended range for product bioequivalence suggested by the FDA. ${ }^{19}$

Caffeine was absorbed rapidly following administration using both gum and beverage with slightly lower exposures achieved after administration using the caffeinated gum compared to the caffeinated beverage (14\% and $9 \%$ lower for AUC and $C_{\max }$, respectively). However, this difference in exposure is likely to be a result of the amount of caffeine made available for absorption from the two matrices, with the $50 \mathrm{mg}$ caffeine dissolved in the beverage being optimally available and easily controllable during preparation by clinical staff, whereas that released by

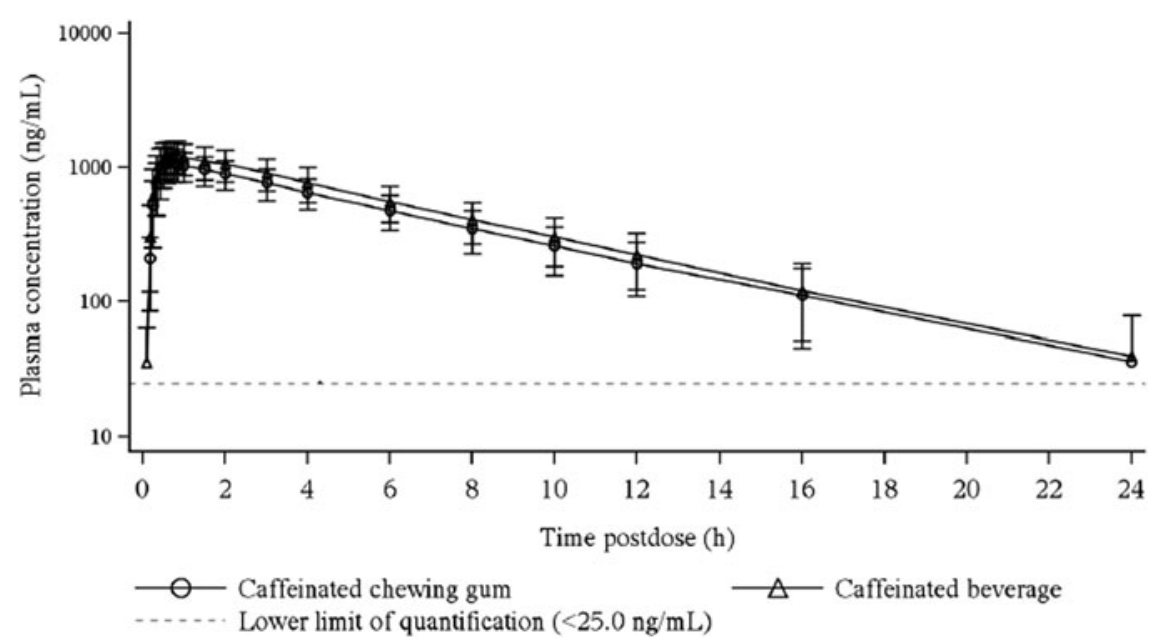

FIG. 2. Arithmetic mean caffeine plasma concentration versus time (through 24 hours) in semilogarithmic scale following administration of a single dose of $\sim 50 \mathrm{mg}$ caffeine using caffeinated chewing gum $(n=15)$ and a marketed caffeinated beverage $(n=16)$ to healthy subjects. 
Table 2. Summary of the Pharmacokinetic PARAMETERS FOR CAFFEINE

\begin{tabular}{|c|c|c|}
\hline Parameter & $\begin{array}{l}\text { Caffeinated } \\
\text { chewing gum } \\
\quad(\mathrm{n}=15)\end{array}$ & $\begin{array}{c}\text { Caffeinated } \\
\text { beverage } \\
(\mathrm{n}=16)\end{array}$ \\
\hline $\begin{array}{l}\mathrm{AUC}_{0-\mathrm{t}} \\
\quad(\mathrm{h} \cdot \mathrm{ng} / \mathrm{mL})\end{array}$ & $6990(33.5)$ & $8100(34.9)$ \\
\hline $\begin{array}{l}\mathrm{AUC}_{0-\infty} \\
\quad(\mathrm{h} \cdot \mathrm{ng} / \mathrm{mL})\end{array}$ & $7440(34.6)$ & $8520(35.2)$ \\
\hline$C_{\max }(\mathrm{ng} / \mathrm{mL})$ & $1180(24.2)$ & $1300(28.6)$ \\
\hline$t_{\max }{ }^{\mathrm{a}}(\mathrm{h})$ & $0.667(0.250-1.50)$ & $0.625(0.333-1.50)$ \\
\hline$t_{\mathrm{lag}}^{\mathrm{a}}(\mathrm{h})$ & $0(0-0.0833)$ & $0(0-0.0833)$ \\
\hline$t_{1 / 2}^{\mathrm{b}}(\mathrm{h})$ & $4.91(1.44)$ & $4.70(1.26)$ \\
\hline$\lambda_{\mathrm{z}}\left(\mathrm{h}^{-1}\right)$ & $0.147(28.8)$ & $0.152(26.7)$ \\
\hline$k_{\mathrm{a}}\left(\mathrm{h}^{-1}\right)$ & $2.99(49.6)$ & $2.72(41.8)$ \\
\hline
\end{tabular}

Geometric mean (CV\%) data are presented for non-DN AUCs and $C_{\max }$

${ }^{a}$ Median (min-max).

${ }^{\mathrm{b}}$ Arithmetic mean (SD).

$\mathrm{AUC}_{0-\mathrm{t}}$, area under the concentration-time curve from hour 0 to the last measureable concentration; $\mathrm{AUC}_{0-\infty}$, area under the plasma concentration-time curve extrapolated to infinity; $C_{\max }$, maximum observed concentration; $t_{\max }$, time of the maximum observed concentration; $t_{\text {lag }}$, time to first quantifiable concentration; $t_{1 / 2}$, apparent terminal elimination half-life; $\lambda_{z}$, apparent terminal elimination rate constant; $k_{\mathrm{a}}$, absorption rate constant.

gum for absorption was reliant on the chewing process. Measurement of caffeine remaining in the discarded gum cud after chewing showed that the mean actual dose of caffeine released by the caffeinated gum, and therefore available for absorption, was $\sim 18 \%$ lower than the intended 50-mg dose. Between-subject variation in the actual dose available from caffeinated gum was high (actual mean dose $40.81 \mathrm{mg}$; SD 3.206) compared to caffeinated beverage (actual mean dose $50.65 \mathrm{mg}$; SD 0.496) and is likely a reflection of the difference in factors such as mastication rate and saliva production between subjects over the 10-minute chewing period, which in turn may influence both the rate and total amount of caffeine release. To account for the differences in actual dose administered between the two caffeine products, the PK parameters were $\mathrm{DN}$ to allow statistical comparison and determine relative bioavailability. This was considered appropriate given that the actual doses achieved were well within the range at which the PK of caffeine has been shown to be dose proportional when administered as a caffeinated beverage ${ }^{12}$ or caffeinated gum. ${ }^{15}$

Although between-subject variation in exposure to caffeine was moderate, the study was carried out in a crossover manner to account for differences in dosing occasion and to allow a within-subject comparison of administration methods.

Based on the ratios of geometric LS means, $\mathrm{DN} \mathrm{AUC} \mathrm{Alt}_{\text {-t }}$, $\mathrm{AUC}_{0-\infty}$, and $C_{\max }$, were $7 \%, 9 \%$, and $12 \%$ higher, respectively, following administration of caffeinated chewing gum compared to caffeinated beverage. However, although the criteria were not set a priori and the study was not statistically powered for concluding bioequivalence, the calculated $90 \% \mathrm{CI}$ fell within the industry accepted bioequivalence limit of $80 \%$ to $125 \%,{ }^{19}$ and therefore caffeine delivered using chewing gum appeared bioequivalent to that delivered using beverage.

Table 3. Statistical Analysis of the Pharmacokinetic Parameters for Caffeine

\begin{tabular}{|c|c|c|c|c|c|c|c|}
\hline \multirow[b]{2}{*}{ Parameter (unit) } & \multirow[b]{2}{*}{ Treatment } & \multirow[b]{2}{*}{$\mathrm{n}$} & \multirow{2}{*}{$\begin{array}{l}\text { Geometric } \\
\text { least squares } \\
\text { means }\end{array}$} & \multirow{2}{*}{$\begin{array}{c}\text { Ratio of } \\
\text { geometric least } \\
\text { squares means } \\
\text { (test:reference) }\end{array}$} & \multicolumn{2}{|c|}{$\begin{array}{c}90 \% \text { CI } \\
\text { for the ratio } \\
\text { (test:reference) }\end{array}$} & \multirow[b]{2}{*}{$\mathrm{p}$} \\
\hline & & & & & Lower & Upper & \\
\hline \multirow{2}{*}{$\begin{array}{l}\mathrm{DN} \mathrm{AUC}_{0-\mathrm{t}} \\
(\mathrm{h} \cdot \mathrm{ng} / \mathrm{mL} / \mathrm{mg})\end{array}$} & Caffeinated chewing gum (test) & 15 & 172 & & & & \\
\hline & Caffeinated beverage (reference) & 16 & 160 & 1.07 & 1.04 & 1.11 & \\
\hline \multirow{2}{*}{$\begin{array}{l}\mathrm{DN} \mathrm{AUC}_{0-\infty} \\
(\mathrm{h} \cdot \mathrm{ng} / \mathrm{mL} / \mathrm{mg})\end{array}$} & Caffeinated chewing gum (test) & 15 & 183 & & & & \\
\hline & Caffeinated beverage (reference) & 16 & 168 & 1.09 & 1.05 & 1.13 & \\
\hline \multirow{2}{*}{$\begin{array}{l}\mathrm{DN} C_{\max } \\
\quad(\mathrm{ng} / \mathrm{mL} / \mathrm{mg})\end{array}$} & Caffeinated chewing gum (test) & 15 & 28.7 & & & & \\
\hline & Caffeinated beverage (reference) & 16 & 25.7 & 1.12 & 1.04 & 1.20 & \\
\hline \multirow[t]{2}{*}{$t_{\max }(\mathrm{h})^{\mathrm{a}}$} & Caffeinated chewing gum (test) & 15 & 0.67 & & & & \\
\hline & Caffeinated beverage (reference) & 15 & 0.58 & -0.08 & -0.17 & 0.08 & 0.3308 \\
\hline \multirow[t]{2}{*}{$k_{\mathrm{a}}\left(\mathrm{h}^{-1}\right)^{\mathrm{a}}$} & Caffeinated chewing gum (test) & 15 & 2.36 & & & & \\
\hline & Caffeinated beverage (reference) & 15 & 2.60 & 0.33 & -0.36 & 1.15 & 0.3894 \\
\hline
\end{tabular}

One subject was excluded from chewing gum treatment since the quantifiable predose value was $>5 \%$ of $C_{\max }$ value. Only paired data were presented and statistically analyzed for $t_{\max }$ and $k_{\mathrm{a}}$. Least-square means and $90 \% \mathrm{CI}$ are based on a linear mixed-effects model with treatment as a fixed effect and subject as a random effect. The ratio and corresponding confidence limits are back-transformed from the original difference and confidence.

${ }^{a}$ Medians, median of differences, approximate $90 \%$ confidence interval and p-value from the Wilcoxon signed-rank test are presented.

DN $\mathrm{AUC}_{0-\mathrm{t}}$, dose-normalized area under the concentration-time curve (AUC) from hour 0 to the last measureable concentration; $\mathrm{DN} \mathrm{AUC}_{0-\infty}$, dose-normalized AUC extrapolated to infinity; DN $C_{\max }$, dose-normalized maximum observed concentration; $t_{\max }$, time of the maximum observed concentration; $k_{\mathrm{a}}$, absorption rate constant; CI, confidence interval; $n$, number of subjects. 
In addition, the actual (non-DN) caffeine plasma concentration versus time profiles were similar for the two caffeine products and there were no statistically significant differences in the $t_{\max }$ or $k_{\mathrm{a}}$. These findings are, in part, supported by those previously reported by Kamimori et al. ${ }^{14}$ who found that at doses of 50 to $200 \mathrm{mg}$, caffeine administered by chewing gum gave a comparable exposure to that delivered by capsule. However, they observed an increased absorption rate with caffeinated gum, which was not observed in this study, and may be due to a slower release of caffeine from a capsule than the beverage used in this study.

The two caffeine products were well tolerated by subjects who participated in this study. No product-related AEs were reported following caffeine administration by either gum or beverage, which was expected given that the dose administered and exposures achieved were well within the published safety limits for single doses.

The intent of this study was to provide initial insights into what, if any, differences in PK profiles occur when similar levels of caffeine are ingested by chewing caffeine out from gum or by drinking a caffeinated beverage. From these data, it could be possible to estimate how to power a study if further and more granular comparison of the PK parameters were desired. One potential source of variability is the mastication rate in the chewing gum group. Differential mastication rates, both within and between subjects, could have contributed to the variation of release rate of caffeine from the chewing gum matrix, and therefore resulting in variation of PK data.

\section{Conclusion}

In summary, the PK profiles of caffeine administered by the chewing gum and beverage were similar, suggesting that the body of scientific literature on caffeine, which is mostly based on data from caffeinated beverages, can be leveraged to support the safety of caffeine delivered by chewing gum. Furthermore, the current maximum safe dose advice for caffeine issued by health regulatory authorities should be applicable whether the dose is administered by chewing gum or oral administration of caffeinated food or beverages.

\section{Acknowledgments}

This study was funded by Mars Wrigley Confectionery. The authors thank Beth Statkus and Edith DeVita of Mars Wrigley Confectionery, Catherine Kwik-Uribe of Mars Symbioscience, David Dinges of the University of Pennsylvania School of Medicine, and Carl Keen and Hagen Schroeter of the University of California-Davis Department of Nutrition for their valuable input on the article. The authors also thank the study participants for their involvement in the study. Becky Jubb and Debra Hidayetoglu of Covance Global Medical and Regulatory Writing provided medical writing support for the article.

\section{Authors' Contributions}

H.C. conducted the clinical trial. All the authors devised the concept and provided interpretation and revisions of the article. All the authors have approved the final version of the article.

\section{Author Disclosure Statement}

P.S., X.P., P.S., and E.M. are employees of Mars Wrigley Confectionery. J.C. is an employee of JBC Consulting. H.C. is an employee of Covance Daytona Clinical Research Unit, Inc.

\section{References}

1. Fredholm BF, Bättig K, Holmén J, Nehlig A, Zvartau EE. Actions of caffeine in the brain with special reference to factors that contribute to its widespread use. Pharmacol Rev. 1999;51:83-133.

2. Wikoff D, Welsh BT, Henderson R, et al. Systematic review of the potential adverse effects of caffeine consumption in healthy adults, pregnant women, adolescents, and children. Food Chem Toxicol. 2017;109: 585-648.

3. Ferré S, Fredholm BB, Morelli M, Popoli P, Fuxe K. Adenosine-dopamine receptor-receptor interactions as an integrative mechanism in basal ganglia. Trends Neurosci. 1997;20:482-487.

4. Ferré $\mathrm{S}$. Role of the central ascending neurotransmitter systems in the psychostimulant effects of caffeine. J Alzheimers Dis. 2010;20:S35-S49.

5. McLellan TM, Caldwell JA, Lieberman HR. A review of caffeine's effects on cognitive, physical and occupational performance. Neurosci Biobehav Rev. 2016;71: 294-312.

6. Cappelletti S, Piacentino D, Sani G, Aromatario M. Caffeine: Cognitive and physical performance enhancer or psychoactive drug? Curr Neuropharmacol. 2015; 13:71-88.

7. Nehlig A. Is caffeine a cognitive enhancer? J Alzheimers Dis. 2010;20:S85-S94.

8. EFSA (European Food Safety Authority). Scientific opinion on the safety of caffeine. EFSA J. 2015;13: 4102.

9. Blanchard J, Sawers SJ. The absolute bioavailability of caffeine in man. Eur J Clin Pharmacol. 1983;24:93-98.

10. Turpault S, Brian W, Van Horn R, et al. Pharmacokinetic assessment of a five-probe cocktail for CYPs 1A2, 2C9, 2C19, 2D6 and 3A. Br J Clin Pharmacol. 2009;68:928-935.

11. Newton R, Broughton LJ, Lind MJ, et al. Plasma and salivary pharmacokinetics of caffeine in man. Eur J Clin Pharmacol. 1981;21:45-52.

12. Bonati M, Latini R, Galletti F, Young JF, Tognoni G, Garattini S. Caffeine disposition after oral doses. Clin Pharmacol Ther. 1982;32:98-106.

13. Cheng WS, Murphy TL, Smith MT, et al. Dosedependent pharmacokinetics of caffeine in humans: Relevance as a test of quantitative liver function. Clin Pharmacol Ther. 1990;47:516-524.

14. Kamimori GH, Karyekar CS, Otterstetter R, et al. The rate of absorption and relative bioavailability of caffeine 
administered in chewing gum versus capsules to normal healthy volunteers. Int J Pharm. 2002;234:159-167.

15. Syed SA, Kamimori GH, Kelly W, Eddington ND. Multiple dose pharmacokinetics of caffeine administered in chewing gum to normal healthy volunteers. Biopharm Drug Dispos. 2005;26:403-409.

16. Shojaei AH. Buccal mucosa as a route for systemic drug delivery: A review. J Pharm Pharm Sci. 1998;1:15-30.

17. McCort-Tipton M. Validation of a method for the determination of caffeine and paraxanthine in human plasma by HPLC with MS/MS detection method validation report, Covance Study No. 2100915; February 15, 2008.

18. Gibaldi M, Perrier D. Pharmacokinetics. 2nd edition. New York, NY: Marcel Dekker, Inc.;1982.
19. FDA Center of Drug Evaluation and Drug Administration. Statistical approaches to establishing bioequivalence. 2001. Available at: www.fda.gov/downloads/ drugs/guidances/ucm070244.pdf. Accessed June 15, 2017.

Address correspondence to: Xiao Pan, PhD, MPH

Mars Wrigley Confectionery 1132W Blackhawk Street

Chicago, IL 60642

E-mail: xiao.pan@effem.com 